\title{
Elements of .Theoretical Aeromechanics*
}

\author{
Part I.-Aerostatics
}

\section{By A. F. Zahm, Ph.D.}

As some knowledge of the science of equilibrium and motion of fluids is essential to the intelligent study of aëronautics, it seems advisable to present a few of the main principles of that science illustrated by practical applications.

Aeromechanics may be divided into two branches aerostatics and aerodynamics. Aerostatics is the science of equilibrium of gaseous fluid; aerodynamics is the science of motion and resistance of such a fluid. The two branches may be treated in succession. We shall begin with aërostatics, or, still better, with the general science of fluid statics.

When the fluid is at rest, or moving with equa velocity at every point, it can offer no resistance to change of shape; that is, it can sustain no shearing force. Its pressure against any surface is, therefore, normal to that surface. From this it easily follows that the pressure at any point of a static ${ }^{1}$ fluid is equal in all directions; for otherwise a short flament of fluid at the point, extending in any two directions of un equal pressure, would move in the direction of th lesser pressure, since the fllament wall can exert no tangential restraining force, being assumed frictionless Hence the term hydrostatic pressure is commonly used for the stress, or force, acting at a point in a material substance, when no tangential or shearing effort is present.

This hydrostatic condition obtains in natural fluids only when they are at rest or moving without change of shape. In perfect or frictionless fluids, which are purely hypothetical, the pressure at every point is hydrostatic, whether the fluid be still or moving without acceleration; but in natural fluids, such as air or water, if there is shearing motion of any kind, there is sure to be friction, either superflcial or internal. This must be reckoned with, and is duly considered in the kinetics of fluids.

For a long time aeronautical investigators believed that the pressure of a gas or of air against.a solid surface is perpendicular, whether the fluid be gliding over the surface or not. But it has been amply proved that when relative motion exists between the surface and fluid there is always surface friction, increasing rapidly with the speed, and hence that the force on the surface cannot be normal.

From the assumption that hydrostatic pressure is normal to a surface follows a second principle, viz, that a pressure applied at any point of a static fluid is transmitted undiminished to all parts of the fluid. For suppose a small, open, cylindrical tube to reach from where the pressure is applied in the fluid to where it is transmitted in any direction taken at random. Since the column of fluid in the tube is at rest, the pressures on its opposite ends must balance, if gravity can be ignored, because they are the only lengthwise force acting on the column, the wall pressure being exactly normal to the length. Hence the pressure at the two points is equal, and the transmission of the pressure is perfect.

As an example of the undiminished transmission of pressure, if a loose-fltting cork in a jug full of wate be struck inward by a sharp blow of the hand the bottom will break out; if a plug be driven into a castiron shell fllled with liquid the shell will burst. Agai the water pressure in a faucet is the same as in the far-away reservoir at the same level, if the water is not flowing. So also the pressure of gas or air is constant at all points at the same level in a system of distributing pipes, if the fluid is at rest and at uniform temperature

The above ignoration of gravity is quite common in engineering, as when computing the pressure of steam in an engine or water in a hydraulic press. But if gravity be taken account of, it is easily seen that the difference of fluid pressure per square unit, at an two levels in the fluid, equals the weight of a vertical column of liquid joining the levels, and of one square unit cross-section.

From the last consideration it follows that a submerged body is buoyed up by a force equal to the weight of the displaced fluid. For the difference of vertical pressure on the ends of any small vertical prism drawn in the body from surface to surface, at different fluid levels, is the weight of a like prism of the fluid; hence for the whole body the difference of

*Abstracted from the writer's forthcoming popular treatise on eromechanics.

1 By static fluid is meant one that is not changing shape or velocity. the whole upward and downward components of pressure on its surface is the weight of the fluid displaced. Or, otherwise, suppose the body replaced by frozen fluid. This has the same surface pressure, and it buoyance evidently equals the weight of the displaced fluid. Whether the body is submerged or buoyed on the fluid surface, the above argument is valid; hence, in general, the buoyance of a body equals the weight of the displaced fluid. This is Archimedes' principle. Evidently the principle is true for a body immersed in a heterogen

Since the surface pressures on any immersed body are the same as on a like body of the frozen fluid whose center of gravity evidently coincides with the center of buoyancy, it follows that the center of buoyancy of any immersed body must be at the centroid of the fluid displaced. The immersed body will, therefore, be in stable, unstable, or neutral equilibrium according as its center of mass is below, above, or a the center of buoyancy. In a common free balloon, for example, in all ordinary positions, the centroid of the entire mass of envelope and load is well below th center of buoyancy; hence the stability is secure.

In general a floating balloon is not at rest vertically, but rises or falls. If the balloon has surplus buoyancy and a slack envelope, it may rise, enlarging in bulk till the envelope is fully distended or till the air is so light that its displaced mass does not exceed the mas of the balloon. Before this altitude of vertical equilibrium it attained, and before the envelope is fully distended, a slight change of mass may cause a great change of level, because the altering volume of the globe nearly compensates the alteration of atmospheri density. Especially is this true if the framing an eargo have relatively slight bulk, as in a sounding ba loon. But it is not well to assume, as some write do, that the strength of the envelope will prevent th gas expanding, and thereby bring about static equilibrium; for the gas so conflned may easily burst the balloon $^{3}$. For this reason, the neck of the envelope be low is usually left open, or feebly closed, in order t relieve undue pressure when the bag is distended.

Simple applications of the foregoing principles a sometimes very useful in designing or understanding air craft. In illustration we may take a few examples of balloon construction. But flrst let it be required to flnd the resultant pressure at the top of a vertical tube full of light gas, if the tube be open below and closed above.

At the open bottom of the supposed tube the pressure of air and gas are equal. At the top the air pressure is less than at the bottom by the weight of a unit square column of air having the length of the tube, while the gas pressure there is less by the weight of a gas column of the length of the tube. Hence at top the gas pressure exceeds the air pressure by the difference in weight of the columns of gas and air. Thus occurs a resultant outward pressure of the gas, which increases from nothing at the bottom to a maximum at the top of the tube, and at any other point is directly proportional to the distance from the bottom of the tube. This increase of outward pressure is, for hydrogen (roughly), 0.08 of a pound per square foot for each foot above the bottom of the gaseous column, near sea level.

From this simple example it follows that the resultant internal pressure in a hydrogen balloon increases one pound per square foot for each twelve feet above any flxed point in the envelope, the pressure at any given level being everywhere the same. It thus appears that if the envelope be very high, or have a long, vertical neck flled with gas, or if a long, unicellular dirigible rears up, the top pressure may become dangerously great. Ignorance or neglect of this consideration ccasionally leads to monstrous and impracticable designs, and sometimes to disaster. Thus a balloon with a long neck has been known to burst at the top, as a cask is burst by pouring in water through a tall, narrow tube. A long dirigible has come to grief by rearing in flight so that the internal pressure caused the prow to explode. Designers of mammoth airships should, therefore, take precautions to ensure their colossal craft against splitting along the back or rearing

2 Centroid means center of mass, which is commonly taken as the center of gravity.

3 Exception is made of the case in which the balloon is made of enormously strong and light material for the express purpose and exploding at the prow as previously stated.

From the foregoing principles, also, can be computed the bursting pressure of an envelope if the unit tensile strength of its fabric is known. If the envelope is spherical the entire internal pressure on the area of the equatorial circle equals the whole tensile strength of the fabric around the circumference. In other words, to flnd the bursting pressure divide the entire circumferential strength by the equatorial area; or, more simply, divide twice the strength of one foot of the fabric by the radius of the sphere in feet. By similar reasoning it can be shown that the bursting strength of a cylinder is half that of a sphere of the same diameter, and that its resistance to splitting is half its resistance to tearing across. Of course, the top pressure in a well-designed balloon can never be allowed to approach the bursting pressure so computed, but a large factor of safety must be allowed.

In addition to the foregoing it may be sometimes desirable to know the strength of a long gas bag regarded as a beam, and subject to forces which tend to double it, as in Santos-Dumont's dirigible ${ }^{4}$ No. 2. The strength in such case can easily be computed in terms of the cross-section and internal pressure. Without giving the detailed proof, the following theorem may be stated; the breaking moment at any section of a round, inflated beam equals the whole internal pressure on the section multiplied by half the radius. ${ }^{5}$

The unit tensile strength of the fabric may be found by direct breakage in a testing machine, or by bursting spheres or cylinders of it by compressed air, due care being taken to measure the internal pressure and diameter at the instant of rupture. But the practice of novices, in which disks of the fabric stretched over the mouth of a tube are ruptured by fluid pressure, may lead to erroneous conclusions, unless the radius of curvature of the fabric at the instant of rupture be accurately determined, and the fabric itself be guarded from shearing, or cutting, at the clamp. If such measurements are correctly made, the tensile strength can be readily computed from the hydrostatic equations of the preceding paragraph.

The buoyancy of the gas in a balloon is the difference between its weight and that of the air it displaces. If it weighs half as much as air, its buoyancy is one half the weight of said air; if it weighs one flfteenth its buoyancy is fourteen flfteenths; if it weighed nothing, its buoyancy would be flfteen flfteenths, which is an increase of one flfteenth over that of pure hydrogen Thus it appears that in point of buoyancy an inflnitely light gas balloon or a vacuum balloon would have no very great advantage, at least for ordinary use. ${ }^{6}$ For sounding balloons and racers a lighter gas might be desirable if it could be retained.

The height to which a given balloon can rise may be found by dividing the total weight by the total volume of the distended balloon and its load, to obtain the density of the air at the level of balanced flotation, then reading in a table the altitude corresponding to that density. In formulating this rule it is, of course, assumed that no considerable weight of gas escapes during ascent to the level of equilibrium; for every wise aeronaut leaves enough slackness in his envelope during inflation to take up the expansion of the gas during the ascent. The proper volume of gas to start with at the lower level must equal the volume at the upper level multiplied by the ratio of the atmospheric densities at the upper and lower levels, assuming that the gas possesses at those levels the temperature of the environing air, which it may do at night or when shaded by a dark cloud.

If the temperature of the gas be assumed equal to that of the environing air, as might ocur in a highspeed metal balloon of good thermal conductivity, it can be shown that the resultant internal pressure, or "net pressure," remains unaltered, however the temperature and barometric pressure of the atmospher may vary. As this theorem is of some interest in the science of dirigibles, the following simple demonstration of it may be presented, as flrst given by the writer in the paper above cited:

In the flrst place, if the atmospheric pressure alone

- When this spindle-shaped balloon lost pressure it first bent hen doubled like a pocket knife.

- A proof of this theorem is given in the present writer's paper, "Some Theorems in the Mechanics of High-speed Balloons"

- For a mathematical proof of the impossibility of floating vacuum. 
alters, while the other elements ${ }^{7}$ remain constant, no change occurs in the net pressure. For the density of the air, and hence the buoyancy of the vessel, changes directly as the barometric pressure; and the vessel will rise or fall to a level where the density of the air is equal to what it was at the flrst position of equilibrium. But if the density at the new position equals that at the old, so also must the atmospheric pressure, since the other elements have not changed. Hence the net pressure is unaltered, which proves the theorem.

Again, if the atmospheric and gas temperatures alter equally, while the other elements remain constant, no change occurs in the net pressure. For the density of the air varies inversely as the temperature; and the vessel will move to a level where the density is the same as at the flrst position of equilibrium. But. if the density at the new position equals that at the old,

7 The other elements of density are the temperature and percentage of moisture. the proportionate increment of air pressure must be directly as the increment of temperature, and therefore equal to the increment of gas pressure. Hence the net pressure remains unaltered, which proves the theorem. Combining the two theorems just established, we obtain the more general one, viz., if the gas temperature of a vertically free balloon keeps pace with the air temperature, no change of net pressure occurs for al. thermometric or barometric changes in the atmosphere. Furthermore, it may be affirmed that if the vessel' bulk varies, while its mass remains constant, practically no variation of net pressure ensues from the bulk change itself. For the pressure increments of the ga and air equal each other, since when the bulk enlarges or contracts the vessel moves to a rarer or denser at mosphere, while the gas pressure changes proportionately, thus leaving the net pressure practically unchanged.

Again, if air is pumped into or out of the balloon, practically no change of net pressure occurs if the bal- loon is free to alter its pressure accordingly, and if the temperatures of the gas and surrounding air remain equal. For changing the weight of air in the hull causes a corresponding change of atmospheric level, and with it a change of external pressure equalling the change of internal pressure. Practically this means that by pumping air into a balloon or its balloonet the vessel can be brought down from any elevation without altering the net pressure. This and the preceding theorem are rigorously proved in the paper previously cited.

The four theorems just proved assert that, in a highspeed metal balloon (which promptly assumes the temperature of the environing air), practically no change of net pressure can occur from any change of density or temperature of the air, from any enlargement or contraction of hull, or from the alteration of weight caused by pumping air into or out of the hull, providing the vessel is free to float to its level of equilibrium. To be continued.

\section{Colloids and Colloidal Solutions*}

\section{Some Examples from Everyday Life}

\section{By Elwood B. Spear}

IF we stir sugar into a cup of water we make what the scientist calls a solution. It is a homogeneous mixture of water and sugar, that is to say, the smallest portion of the liquid that we can see even under the most powerful microscope contains relatively the same amount of sugar and water as any other portion of the solution. According to the modern theories of the constitution of matter, both water and sugar are present as molecules. These molecules are too small to be seen by the eye even aided by the microscope, and the whole sclution, therefore, appears to us to be made up of only one substance. Solutions where the single molecules of the dissolved substance move freely about among the molecules of the solvent are called crystalloid solutions, molecules of the solvent are called crystalloid solutions,
and the dissolved substance is called a crystalloid. These solutions may be colorless like white sugar dissolved in water or colored like strong tea, but they are always clear and more or less transparent.

Colloidal Suspension.-The water in our rivers and streams in early spring is almost always turbid. This is due to the fact that it contains large quantities of nud and other substances that are divided into very small particles. These particles are held in suspension by the water because their weight is so nearly equal to that of the same volume of water that the whole mas must remain quiet before the mud particles can settle down to the bottom. If the water is moving the mud varticles are carried along with it, and the settling out is prevented by the mixing action of the running water If muddy water is allowed to stand, we notice that the largest particles fall out in a few minutes, while it usually takes hours before the water is clear of all the very small particles. This is a general law that for a very flnely divided substance suspended in a liquid the larger the particles, the faster they will fall to the bot tom. It is possible to obtain particles so small that they do not fall out for several months, and these particles have been given the names of "colloids," whil the whole solution is called a "colloidal suspension."

Some of these colloidal particles contain a few, some of them many hundreds of molecules of the dissolved subtance. the liquid and moves as a whole, just as eleven individual players form a football team and make a concerte attack on the opponent's goal, or a thousand soldier form a regiment and charge a fort.

Colloidal suspensions can be made of many metals, such as iron, silver, gold and platinum, by allowing an electric current to jump across through water between the points of two wires of the metal in question. The particles of a colloidal suspension of platinum made by this process are of various sizes, some of them large enough to be seen with the unaided eye, while others are not much larger than water molecules. Some of the large particles fall out in a few hours, while the extremely small ones may remain suspended in the water for years.

Size of Colloidal Particles.-Suppose one of these par ticles and the head of a pin were each enlarged in the same proportion until the particles could be seen by the human eye, the head of the pin would then appear a a huge mass of metal as large as a seven-story building. True Colloids.-If we attempt to dissolve a small piece of jelly in warm water we obtain a solution that appears to be clear. In reality the molecules of the jelly are not single and independent of each other, but have formed groups of two's, three's, ten's, etc., lik school children at intermission. We have made here a true colloidal solution, which differs from colloidal sus pensions chiefly in the fact that particles are soft an plastic, resembling jelly, soap, rubber, etc., while those of the suspensions are much harder, like tiny pieces of metal.

Blood a Colloidal Solution.-Most people imagine that the blood is a solution like red ink where every portion of the liquid, however small, is the same color. In point of fact, however, the red color is due to the presence of innumerable small red particles called corpus-

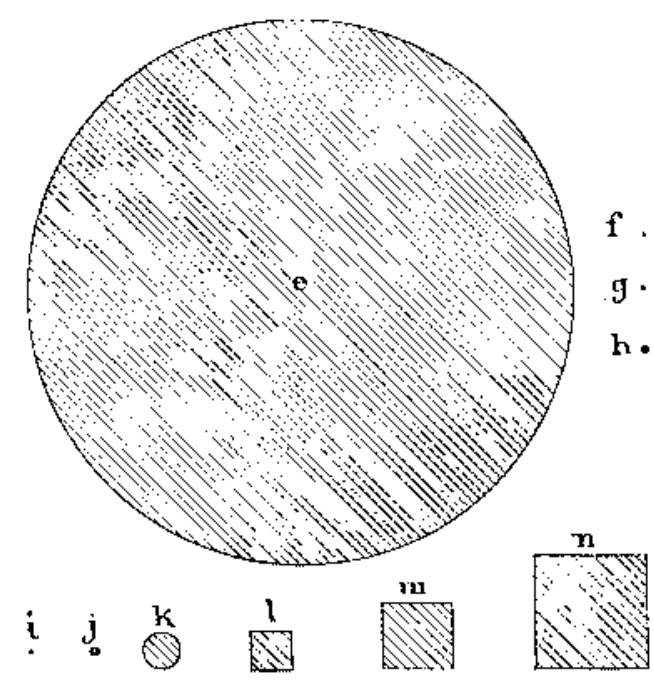
In the above figure, e represents a blood
corpuscle enlarged 7,000 times, and $f$. g and
$h$ the comparative size of the particles in a colloidal suspension of gold. Now conside that $f, g$ and $h$ are enlarged to $l, m$ and $n$ then $i, j$ and $k$ will represent the comparative size of molecules of alcohol, chloroform and starch, respectively

cles, floating about in a waterlike liquid. These are large enough to be seen by a powerful microscope. In addition to the red particles there is also a conside

Mill.-Milk also is a most interesting colloidal solution containing yellow and white particles. If milk is allowed to stand, the yellow particles unite and float on top, and we call them cream. When the milk sours the white particles unite and we get thick milk. Cream, however, contains both white and yellow particles, because when it is churned we get the yellow particles in the form of butter, while the white ones remain in the buttermilk. If now the buttermilk is allowed to stand, we flnd that the white particles have united and fallen to the bottom, while the clear amber-colored liquid is left on top.

Colloidal Particles Grow.-The colloidal particles of a gold solution may be caused to unite together and grow larger by violent stirring, just as churning will gather the yellow particles of cream to form solid butter. This uniting of the colloids to form larger particles is called "coagulation," and is produced most easily by violently stirring a hot solution. Some of our readers will remember in boyhood days when obliged to churn how delighted they were when the cream became too warm, because the butter came faster; in other words, the heat had done a part of the churning.

The reason that the flow of blood from a wound can be stopped is because the red particles under the action of the air unite or coagulate and stop up the wound and thus prevent further loss of blood. This is spoken of as the "clotting" of blood.-Science Conspectus.

\section{The Effects of Electric Currents on Reinforced} Concrete

ALA Rming news has been published repeatedly of late years on the destructive effects supposed to be exerted by electric currents on concrete and iron concrete. Some experimenters have even asserted that blocks of concrete submitted to a relatively weak current might become loosened sufficiently in their structure to be cut with a knife.

Now a German magazine, Die Bauvelt, has addressed a circular inquiry to the experts and engineers of all Germany, with a view to ascertaining whether any insufficiently insulated electric conductors had ever given rise to prejudicial effects on iron concrete constructions. From this inquiry it appears that not a single case of electric currents having in any way endangered a concrete building or some of its parts is so far on record in Germany. The data collected related to a great number of power houses as well as iron concrete masts and all sorts of private houses. Even iron concrete water towers which by their isolated positions and the water contained in their interior, would be particularly exposed to destructive effects, have never shown tho least trace of destruction due to the action of electric currents.

It will be remembered that in connection with Knudson's experiments in 1908, a series of concrete and iron concrete blocks were submitted to high pressure in fresh water or sea water, those kept in fresh water being found to be crushed more easily under the action of electric currents than blocks immersed in salt water. On the other hand experiments made in 1910, by W. Gehlet, have shown the prolonged action of strong electric currents to result in desiccation not only of concrete blocks previously kept in water but even of those stored in a dry place. Rammed concrete, however, undergoes an appreciable reduction of its resistance to pressure. The vaporization of the moisture of concrete under the action of electric currents, however, takes place only under greatly exaggerated conditions as compared with those occurring in actual practice. The richer the concrete mixture, the more rapid will be the process of desiccation, while a poorer mixture, on the other hand, has a higher initial strength.

In the case, however, of iron concrete blocks, experiments show; under the action of electric currents, the formation of flssures at the positive electrode. The surface of the iron armature forming the positive pole is covered entirely with rust, whereas a negative iron electrode remains perfectly smooth and the concrete $a b-$ solutely intact. When using brass electrodes in the place of iron ones no harmful action is observed.

Incidentally, these experiments show concrete to be conductor "of the second class," its electrical resistance decreasing as the temperature increases and inversely.

Preserving Furs and Woolen Clothing.-Thymol powder has been recommended as one of the best preservam tives for furs and woolen clothing. The articles should be sprinkled with the powder and wrapped in paper and then put by in tight boxes.-Cosmos. 